\title{
Effect of structure period and stiffness ratio on seismic performance of structure with additional metal dampers
}

\author{
Sun jianjie $e^{1, a}$ \\ ${ }^{1}$ School of Civil Engineering and Architecture, Jiangsu University of Science and Technology, \\ Zhenjiang Jiangsu, 212003, China.
}

a304230515@qq.com

Keywords: Metal dampers; Equivalent damping ratio; Stiffness ratio; Damping effect

Abstract: The energy dissipation structure is set up in certain parts as energy dissipation device, which consumes most energy caused by the earthquake to reduce the seismic response of structures. Based on the study of additional metal dampers and generating calculation method of equivalent damping ratio, vibration differential equations and computer programs are established. Taking damping ratio $f_{k}$ as a parameter and structure period $T_{f}$ as a independent variable for elastic-plastic time history analysis, change rules of the particle displacement and the absolute acceleration response value are discussed. Thus the following conclusions are obtained: (1) The smaller structure period and the larger Stiffness ratio will lead to the better displacement damping effect. (2) When $f_{k}$ is at the range of 1 to 6 , acceleration response value decreases and gains the smallest value as $f_{k}$ is 4 ; when $f_{k}$ is at the range of 7 to 10 acceleration response value does not decrease all the time.

\section{Introduction}

In recent years, the loss caused by the earthquake is more and more serious, people have gradually realized that the importance of control the disaster caused by the earthquake. Through the study scholars found reasonable and effective way is adding control device to the structure to compose energy dissipation structure. Dampers consume most of the energy from the earthquake, thereby reducing the seismic response of structures ${ }^{[1]}$. In this paper, install a metal single-mass damper system as the research object, vibration differential equations and computer programs are established for elastic-plastic time history analysis. Comparison the seismic response of energy dissipation structure and original structure, to study the energy dissipation structure damping performance.

\section{Analysis model}

The research object of this paper is the energy dissipation structure with additional metal damper, Figure 1 is additional metal damper structure diagram; Figure 2 is additional metal dampers structural analysis model. Where in the 1-2 segment represents additional system consisting of a metal damper and support, $k_{d}$ represents a metal damper elastic stiffness, $k_{b}$ represents the stiffness of support, $k_{a}$ represents elastic stiffness of additional system, its size is ${ }^{[2]}$ :

$$
k_{a}=\frac{1}{1 / k_{d}+1 / k_{b}}(1)
$$

3-4 segment represents the main part of the original structure, $k_{f}$ represents the elastic stiffness of the structure; damping 5-6 segments indicates the original structure, $c$ represents the damp of original structure, $m$ represents the mass, the total stiffness of the structure with additional damper is

$$
k=k_{a}+k_{f}
$$




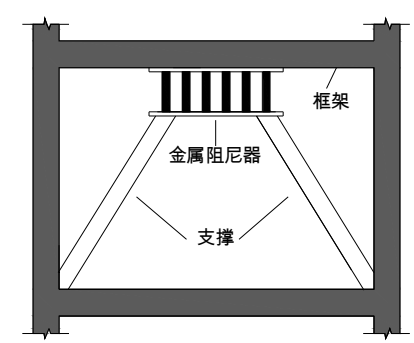

Fig.1 Additional metal damper structure diagram

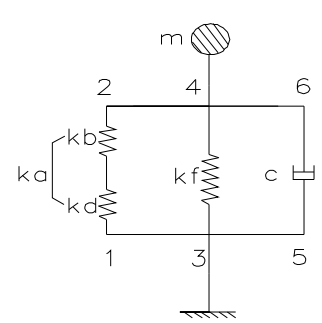

Fig.2 Additional metal dampers structural analysis model

\section{Equivalent viscous damping ratio}

When structure go into the plastic state, the area enclosed by hysteresis curve equal to the energy absorbed by structure from seismic force. In the actual design, it is impossible by the hysteresis curve area calculate the equivalent viscous damping ratio; this article will use another method to calculate the equivalent damping ratio ${ }^{[3]}$ :

$$
h_{e q}=h_{0}+\frac{2}{\mu \pi p} \ln \frac{1+p(\mu-1)}{\mu^{p}}(3)
$$

$p$ is calculated using the following formula

$$
p=\frac{1}{1+k_{a} / k_{f}}(4)
$$

Suppose the original structure is always in an elastic state, and the damper into the plastic state. Using formula (3) to calculate the equivalent damping ratio.By changing the stiffness ratio and ductility factor, to observe the equivalent damping ratio $^{[4]}$, and obtain the following conclusions:

Figure (a) and (b) shows that when $\mu$ unchanged, $h_{e q}$ increases with the increasing of stiffness ratio, when the stiffness ratio unchanged, $h_{e q}$ increases with the increasing of $\mu$.From the figure we can see the best ductility factor ranging from 4-8.

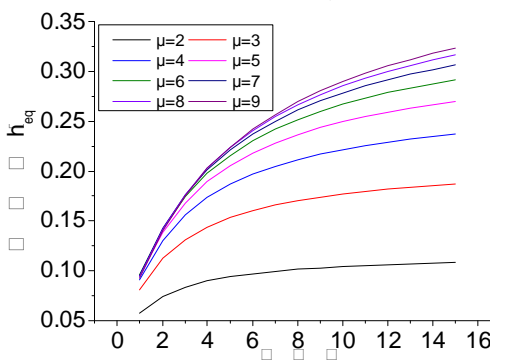

(a) Relationship between $h_{e q}$ and $k_{d} / k_{f}$

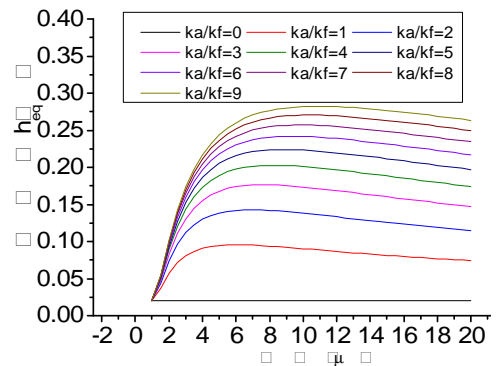

(b) Relationship between $h_{e q}$ and $\mu$

Fig.3 Equivalent damping ratio

\section{The example model}

In this paper, single-layer steel frame structure is used for the study object. Suppose mass distribution is $1000 \mathrm{k}_{\mathrm{g}} / \mathrm{m}^{2}$, then the Quality of single-layer structure is $\mathrm{m}=1200000 \mathrm{~kg}$. The cycle of original structure is taken as $T_{f}=0.1 \mathrm{~s}-3.0 \mathrm{~s}$. The stiffness of metal damper according to the elastic stiffness ratio $f_{k}=0-10$. ( $f_{k}$ is the ratio of damper stiffness and rigidity of the original structure). Additional support stiffness is $k_{b}=1.2 \times 10^{11} \mathrm{kN} / \mathrm{m}$, Suppose the structure is always in an elastic state, and the damper into the plastic state. The original structure is linear elastic restoring force model, the damper is an ideal elastoplastic restoring force model(Plastic stiffness is 0)Restoring force of study model as follows: Elastic stiffness calculated by $T_{f}=2 \pi \sqrt{m / k_{f}}$.The first yield stiffness sk1 taken as elastic stiffness structure. The first yield displacement taken as $u p_{1}=0.01 \mathrm{~m}$. 


\section{The results of time history analysis}

In this paper, the elastic stiffness ratio $f_{k}$ as a parameter, the original structure period is variable, to elastic-plastic time history analysis, analysis of energy dissipation structure displacement reaction, acceleration response. The value of the original structure period $0.1 \mathrm{~s}-3.0 \mathrm{~s}$, elastic stiffness than taking $0-10, T_{f}$ is original construction period, $T_{0}$ is the cycle of energy dissipation structure. In this paper, Art hachinohe wave, Art kobe EL wave and wave, Hachi wave are used to analysis. And compare the displacement response and acceleration response of the structure. Peak of ground acceleration is $400 \mathrm{~cm} / \mathrm{s}^{2}$, the time interval is $0.01 \mathrm{~s}$, the role of time is $50 \mathrm{~s}$.

\section{Displacement reaction}

In fig.4, the abscissa represents the original structure period, the ordinate represents the displacement reaction, parameter is stiffness ratio $\mathrm{fk}$. In fig.5, the abscissa represents the original structure period, the ordinate represents displacement ratio $\gamma$, parameter is stiffness ratio $f_{k}$.

As shown in the figure the greater stiffness of the damper, the smaller displacement reaction of energy dissipation structure, damping effect is more obvious. With the increase of $f_{k}$, displacement reaction of energy dissipation structures are less than the displacement of the original structure, namely additional metal damper structure has good shock effect.

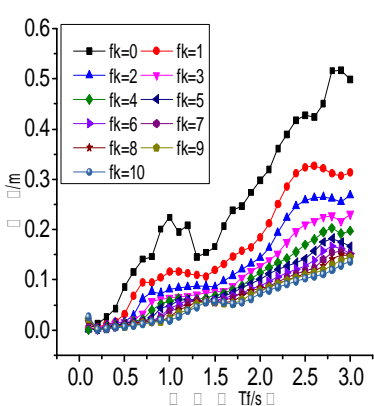

(a)EL wave

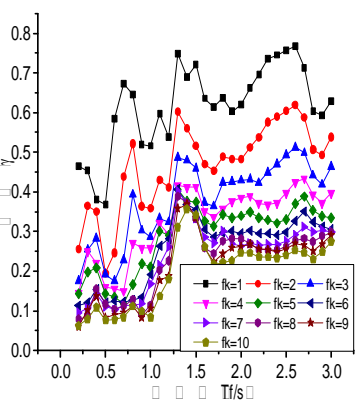

(a)EL wave

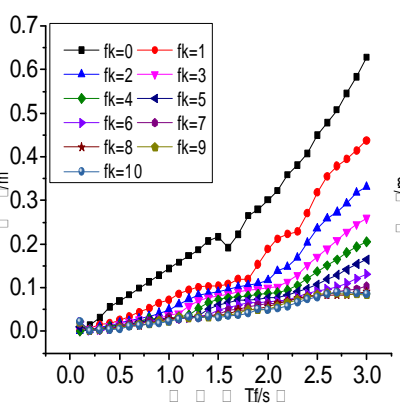

(b)Art hachinohe wave

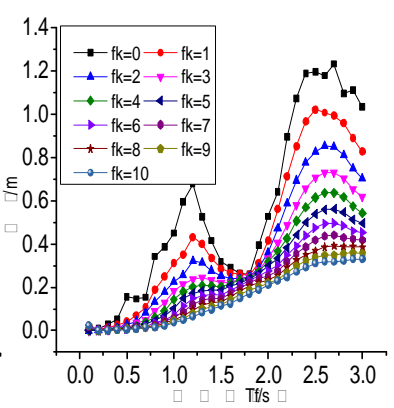

(c)Hachi wave

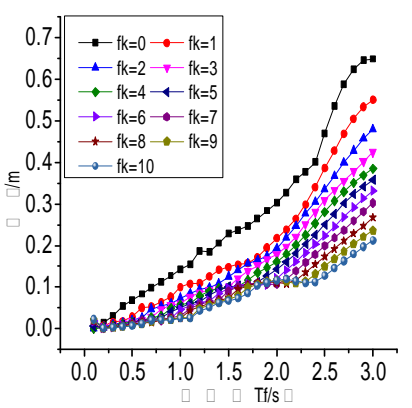

(d) Art kobe wave

Fig.4 Displacement response

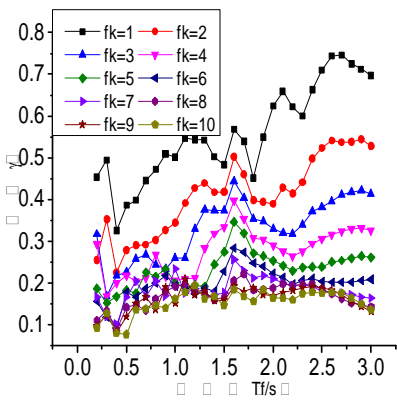

(b) Art hachinohe wave

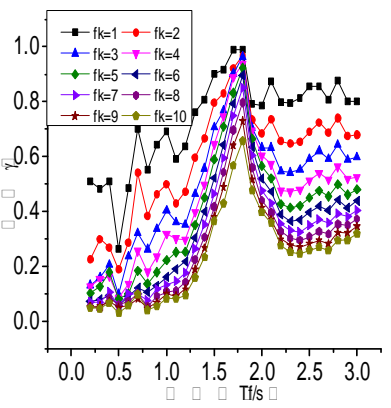

(c)Hachi wave

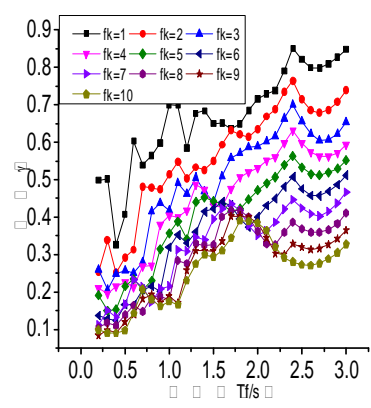

(d) Art kobe wave

Fig.5 Displacement response ratio

\section{The acceleration response}

In fig.6, the abscissa represents the original structure period, the ordinate represents the acceleration reaction, parameter is stiffness ratio $f_{k}$. In fig.7, the abscissa represents the original structure period, the ordinate represents acceleration ratio $\gamma$, parameter is stiffness ratio $f_{k}$.

By the following figure shows the acceleration response is related with cycle of original structure and the stiffness of the damper. when the value of $f_{k}$ from 1 to 6 , the acceleration response value of additional damper structure can be reduced, and when the stiffness ratio $f_{k}$ is 4 , 
acceleration value is least. but when the value of $f_{k}$ from 7 to 10 , the acceleration response values were not fully reduced.

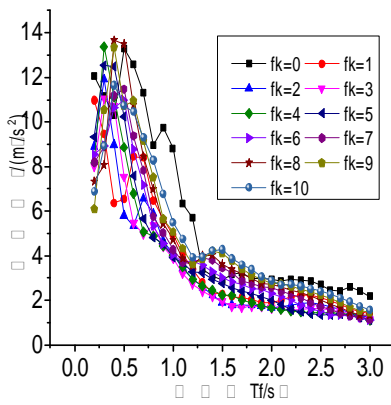

(a)EL wave

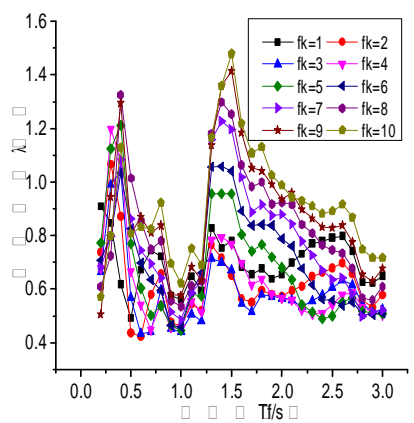

(a)EL wave

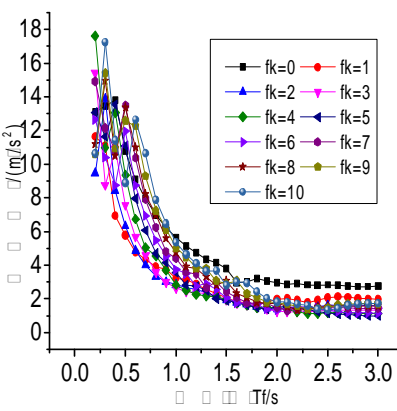

(b)Art hachinohe wave

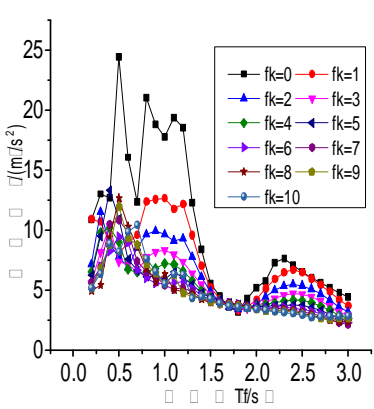

(c)Hachi wave

Fig.6 Acceleration response

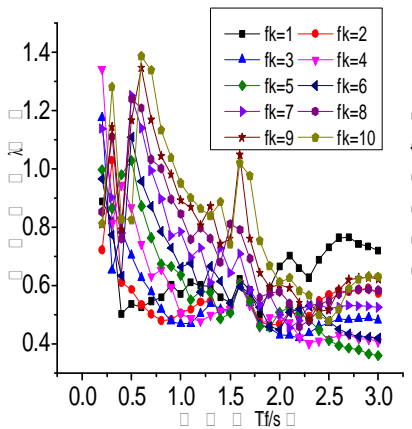

(b)Art hachinohe wave

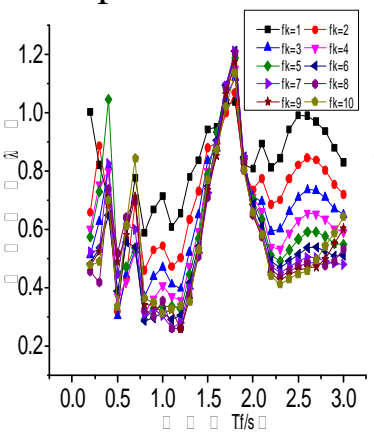

(c)Hachi wave

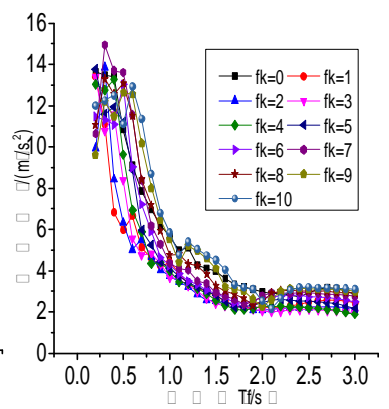

(d) Art kobe wave

Fig.7Acceleration response ratio

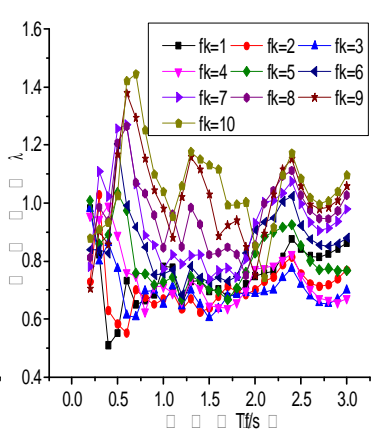

(d) Art kobe wave

\section{Conclusions}

In this paper, based on the study of energy the dissipation structure with additional metal dampers. Consider the calculation method of equivalent damping ratio, vibration differential equations and computer programs are established, Taking stiffness ratio $f_{k}$ as a parameter and structure period as a independent variable for elastic-plastic time history analysis. The displacement and the acceleration response are discussed, the following beneficial conclusions are obtained:

(1) The smaller structure period and the larger Stiffness ratio will lead to the better displacement damping effect.

(2) When $f_{k}$ is at the range of 1 to 6 , acceleration response value decreases and gains the smallest value as $f_{k}$ is 4 ; when $f_{k}$ is at the range of 7 to 10 acceleration response value does not decrease all the time.

\section{References}

[1]Zhang min. Building seismic analysis and vibration control.[M].Chendu: Southwest Jiao tong University Press,2007:174-179

[2]Japan's isolation structure Association. Passive damping structural design and construction manual.[M].Beijing: China Building Industry Press, 2008:15-20.

[3]Lu Weidong, Liu Weiqing, Wang Tao. Calculation method for additional equivalent damping ratio of energy dissipation structure [J]. Journal of Nan jing University of Technology,2009 31(1):97-100.

[4]Pei Xingzhu, He Fangqian. Research on the design method of energy dissipation structure.[J]

Building Structure ,2013,43(4):60-65. 